\title{
Aspectos clínicos y técnico a considerar en los controles de las prótesis parciales removibles.
}

Clinical aspects and technician to considering

in the controls of partial removable Prótesis.

Fecha de Recepción

04 de julio de 2012
Aspectos clínicos e técnico a considerar nos controles das Prótesis parciais removibles.

\section{Laura Huber \\ Auxiliar Docente Primera Categoría.}

María Claudia Gallego

Magister. Jefe de Trabajos Prácticos. Cátedra Clínica de Prótesis I Curso. Codirector de Proyecto.

Roque Oscar Rosende Profesor Titular. Cátedra Cirugía I Técnicas Anestésicas. Director de Proyecto.

\section{Lugar de trabajo Facultad de Odontología. Cátedra Clínica de Prótesis I Curso. Universidad Nacional del Nordeste. \\ Campus Deodoro Roca.}

Aceptado para su publicación 16 de diciembre de 2012

\section{Resumen}

La anodoncia parcial forma parte de la realidad social de nuestro medio, una PPR bien diseñada puede actuar funcionalmente, son estéticamente aceptables, de larga duración sin dañar las estructuras de soporte. Tiene un costo razonable, ofrece restauraciones cómodas, eficientes y manteniendo el contacto oclusal, conservando los pilares libres de caries y enfermedad periodontal. Se verifica el buen funcionamiento a través de controles a distancia para evitar problemas que surgen del uso incorrecto, de un mal mantenimiento y del inadecuado mantenimiento de las conductas higiénicas.

La importancia de implementar los controles posteriores a la instalación permite proteger los dientes remanentes y el estado periodontal, evaluar la integridad y buen funcionamiento de todos los componentes que forman la PPR.

\section{Palabras Claves}

Integridad, Mantenimiento de la prótesis, cromo cobalto, prótesis dental.

\section{Abstract}

Partial anodontia is part of the social reality of our environment. A properly designed RDP may act functionally, it is aesthetically acceptable, long lasting and it does not damage the supporting structures. It also has a reasonable cost and it offers comfortable and efficient restorations which maintain occlusal contact and keep the foundations free of decay and periodontal 
disease.

Its proper functioning is verified through the use of remote controls to avoid problems arising from misuse, poor maintenance and inadequate hygienic behavior.

The importance of implementing post-installation checks is that this action not only allows to protect the remaining teeth and the periodontal status, but also to assess the integrity and the smooth functioning of all components that form the RDP.

\section{Key Words}

Integrity, prosthesis maintenance, chrome cobalt, dental prosthesis, removable dental prosthesis.

\section{Resumo}

A anodoncia parcial faz parte da realidade social de nosso médio, uma PPR bem desenhada pode actuar funcionalmente, são esteticamente aceitáveis, de longa duração sem dañar as estruturas de suporte. Tem um custo razoável, oferece restaurações cómodas, eficientes e mantendo o contacto oclusal, conservando os pilares livres de caries e doença periodontal.

Verifica-se o bom funcionamento através de controles a distância para evitar problemas que surgem de uso incorrecto, uma má manutenção e da inadecuada mantención das condutas higiénicas. A importância de implementar os controles posteriores à instalação permite proteger os dentes remanentes e o estado periodontal, avaliar a integridade e bom funcionamento de todos os componentes que formam a PPR.

\section{Palavras Chaves}

Integridade, Manutenção da prótesis, cromo cobalto, prótesis dental.

\section{Introduccion}

La integridad del cuerpo humano es la base para que pueda existir una adecuada funcionalidad de los órganos y sistemas, la boca no es la excepción. El contar con las piezas dentales en buena condición es importante no solo para la función masticatoria y sino también en la nutrición, en la estética, en el mecanismo de lenguaje y en la calidad de vida.

La conservación de cada órgano dental es vital para el funcionamiento del sistema estomatognático y a pesar que los porcentajes de personas edéntulas tienden a aumentar, se recurre con frecuencia a la prótesis dental para lograr rehabilitar estos pacientes.

Actualmente la anodoncia parcial forma parte de la realidad social de nuestro medio, una prótesis parcial removible (PPR) diseñada y elaborada correctamente prueba que este tipo de aparatología puede actuar funcionalmente, son estéticamente aceptables y de larga duración, sin dañar las estructura de soporte, permite un tratamiento a un costo razonable, ofrece restauraciones cómodas y eficientes con soporte adecuado y manteniendo las relaciones de contacto oclusal, conservando los pilares libres de caries y enfermedad periodontal permitiendo que la PPR sea un tratamiento duradero ${ }^{1-2}$.

Para comprobar el buen funcionamiento de la PPR es necesario realizar controles a distancia para evitar problemas que puedan surgir por el uso incorrecto, por un mal mantenimiento y por la falta de las conductas higiénicas.

El paciente debe conocer la última fase del tratamiento que son los controles periódicos y su importancia radica en evitar el uso descuidado de la prótesis, proteger los dientes remanentes y el estado periodontal, con la establecimiento de medidas higiénicas adecuadas para las piezas dentarias y para el dispositivo protésico instalado.

\section{Desarrollo}

La PPR forma parte de la prostodoncia, rama de la Odontología que se ocupa de la restauración y el mantenimiento de la función oral, el bienestar, el aspecto y la salud del paciente mediante la restauración de los dientes naturales y/o la restitución de los diente perdidos con sustituto artificial ${ }^{3}$. Este tipo de dispositivo cumple con los principios básicos de la prostodoncia, permite eliminar la patología, preservar la salud de los dientes y de estructuras orales, restaurar la función oral con comodidad y la estética sin interferir con la fonética.

El conocimiento de los componentes de la PPR y sus características principales, es fundamental para la evaluación clínica en el momento de realizar los controles para poder verificar el estado y funcionamiento de los mismos.

La estructura colada de la PPR está compuesta 
por una serie de elementos que deben ser diseñados para cumplir con la función de conector mayor, conectores menores, apoyos oclusales, retenedores directos e indirectos base y dientes artificiales todos dependiendo del diseño funcional de la PPR ${ }^{3}$.

El conector mayor tiene como función la conexión de las partes de la PPR. Su principal característica es la rigidez que evita que no se flexione durante los movimientos masticatorios y durante la transmisión de fuerza. La falta de rigidez genera fuerzas lesivas en los dientes pilares y los demás componentes del aparato protésico ${ }^{4}$.

La Barra Lingual es el conector mayor más utilizado, en una relación de $2: I$ con respecto a la Placa Lingual, siendo aquel el conector mayor de elección ya que contribuye al mantenimiento de la salud periodontal. La utilización de Placa Lingual con la implementación de buena higiene bucal mantiene sanos los tejidos y no hay efectos perjudiciales en la parte de la cubierta por metal. Siempre en Clase I y II debe procurarse una buena retención indirecta.

En cuanto a conector mayor superior más utilizado es banda palatina y probablemente se debe al menor cubrimiento del paladar y por resultar más cómodo para el paciente. Generalmente es utilizada en vía de carga dentaria y mixta dentosoportadas. La placa palatina, considerada como el conector de elección para los casos que exista reborde alveolares amplios, y en vía de caga mixta mucodentaria o mucosa.

Los conectores menores deben ser rígidos y adaptarse a los espacios interdentarios, para cumplir su función de transferir las cargas funcionales recibidas a los dientes pilares, transmitir las fuerzas aplicadas a todos los elementos de las prótesis.

Los retenedores son los encargados de mantener la PPR en su posición, pueden ser directos e indirectos según el lugar donde esté actuando la fuerza de desplazamiento. El retenedor directo utilizado con más frecuencia es el gancho circunferencial, debido a que este admite variaciones y se puede emplear en dientes superiores o inferiores, es el retenedor más sencillo de diseñar, construir y reparar, por su rigidez y forma de acceso oclusal, no se deforma fácilmente y presenta excelente soporte y estabilidad horizontal. El inconveniente más observable es la visibilidad del brazo retentivo.
En un diseño de PPR a extensión distal se emplea generalmente el gancho RPI, por que libera de tensiones al diente pilar $y$ ejerce menor concentración de fuerzas en las estructuras de soporte. El retenedor combinado Colado - Forjado es otro tipo de retenedor utilizado con frecuencia, por que presenta una buena distribución de tensiones sobre las estructuras de soporte del diente pilar y cumple con los requisitos del diseño, sin embargo, es un retenedor de tipo circunferencial donde el control de la cobertura dental y el compromiso gingival no es beneficioso.

Los apoyos que descansan sobre una superficie dental preparada, tiene por objeto dar soporte vertical, evitando daños sobre la mucosa y la encía. Son realizados por mesial o distal de los premolares y molares y cuando se colocan sobre los dientes del grupo anterior se realiza en el cíngulo de caninos. En un estudio In vitro 5 se ha determinado el espesor mínimo necesario para que los apoyos cumplan su función. En las aleaciones de cromo cobalto es de $2 \mathrm{~mm}$ de profundidad y el grosor deberá ser al menos de I,03mm como mínimo. Una disminución en estas dimensiones produciría el aumento de tensión en el metal promoviendo la desaptación y/o fractura del apoyo.

La colocación de apoyos en el sector anterior es generalmente dificultoso en el caso que el diente pilar no sea el apto, es decir poseer su corona clínica con amplias restauraciones, en estos casaos se preferirá la utilización de incrustaciones o coronas $^{6}$.

Los planos guías preparados en la pieza dentaria vecina a una brecha guían la trayectoria de inserción y remoción de la prótesis. El aditamento metálico colado para alojarse y acoplarse en dichos espacios cumple la función de retención, estabilización horizontal, disminución del socavado proximal, estética y reciprocidad. Un estudio In vitro 7 acerca del papel del plano guía, determina la importancia del cumplimiento de las funciones y examina la resistencia al desplazamiento ejercida durante la inserción y remoción. Concluye que la retención ejercida por fricción es fundamental para la retención indirecta de la PPR.

La base o silla de la PPR permite trasmitir y absorber las fuerzas generadas durante la oclusión. Es indispensable que se adapten perfectamente a los rebordes residuales para evitar lesionarlos. La 
estabilidad de la base no genera sobrecarga en el diente pilar y las fuerzas se distribuyen de forma homogénea sobre los tejidos de la cresta alveolar, lo que evita a su vez la reabsorción del proceso alveolar. Para lograr una base estable es necesaria una réplica precisa de la zona de soporte de la prótesis. Este principio de base estable reduce al mínimo el movimiento de la prótesis bajo carga y se podrá lograr el éxito a largo plazo. La diferencia de movimiento bajo carga es más acusada en prótesis con una adaptación deficiente ${ }^{89}$.

Una estructura colada es capaz de otorgar soporte, retención y estabilidad a la PPR, ofreciendo un rendimiento funcional que está en relación con las propiedades de la aleación metálica. Las aleaciones para prótesis dental se introdujeron en la década de 1930 siendo la aleación de cromo-cobalto la más usada ${ }^{10}$.

La demanda de uso de esta aleación ha ido aumentando de forma progresiva. El cobalto es el elemento fundamental de la aleación, le confiere dureza, resistencia y rigidez. Tiene un alto punto de fusión y rápido endurecimiento mecánico, que a la hora de evaluar su rendimiento constituye un inconveniente para la aleación, dando lugar a la fractura de los ganchos por enfriamiento .II El Cromo le confiere a la aleación resistencia a la corrosión y a la pigmentación y disminuye el punto de fusión.

La popularidad de esta aleación radica en su baja densidad (peso), buena conductividad térmica, elevado modulo de elasticidad (rigidez), bajo costo, resistencia a perder el brillo, exactitud dimensional de colado, pulido final, disponibilidad y versatilidad de la aleación.

Al colar aleaciones de cromo-cobalto se pueden formar porosidades sub superficiales y otros defectos como concavidades internas de retracción, contracciones filamentosas, oclusiones gaseosas en forma de porosidad, grandes oquedades por humedad de los moldes o por gas del propio metal fundido. Por estas razones, es muy importante extremar las precauciones y cuidar todos los detalles técnicos de colado.

Un estudio clínico radiográfico ${ }^{12}$ señala posibles defectos estructurales existentes en las estructuras coladas de PPR. Sus resultados reportan que uno de cada diez ganchos evaluados presenta defectos en los puntos de unión de los brazos con el conector. El elemento que presenta más fallas es el conector mayor en un $\mathbf{4 2} \%$ los cuales fue visualizado a simple vista solo un $25 \%$ de ellas. Rivas concluye que es importante verificar la integridad del armazón colado mediante la evaluación radiográfica en la detección de fallas en los diferentes elementos de la PPR la cuales no pueden ser detectadas a simple vista.

Existen estudios que relacionan la rigidez del armazón colado con el tipo de diseño y como afecta a los tejidos de soporte. Determina el efecto que produce la rigidez, el grosor y la forma de los conectores mayores maxilares. Para ello se puso a prueba cinco diseños de conectores mayores sobre un mismo modelo maestro. Estos armazones eran de diferente ancho y grosor, al que sometieron a una máquina de prueba con aplicación de fuerzas verticales (torsional) y horizontales (compresivas). Los resultados arrojaron que el conector mayor en forma de placa palatina fue el más rígido y soporta mejor las cargas torsionales y el tipo de diseño banda antero posterior es el más eficaz para soportar las fuerzas compresivas $^{13}$.

Estudios comparativos de los efectos del conector mayor maxilar tipo banda palatina antero posterior y placa palatina sobre los dientes pilares. Estos fueron colocados sobre modelos y puesto a prueba de cargas y fotografiados con luz polarizada para observar la descarga de tensión. Los resultados arrojaron que las placas palatinas descargan su tensión sobre las regiones del paladar duro y los dientes pilares sufren menos tensión, en cambio las bandas palatinas antero posterior descargan su tensión sobre los dientes pilares.

Otro tema a tener en cuenta es el pulido final del armazón metálico para las PPR es un requisito fundamental. Así estos autores recalcan la importancia del pulido pero también advierten que un pulido excesivo o impropio de estos metales trae como consecuencia zonas de reducción de metal que atentan con la rigidez estructural de esta zonas ${ }^{14}$.

\section{Importancia de los Controles Posteriores}

La responsabilidad por el éxito final de la PPR es compartida por el odontólogo y el paciente. El reto más significativo para el paciente y el odontólogo es implementar los conceptos de educación para el uso del dispositivo. Otra circunstancia es la falta aceptación para este tipo de rehabilitaciones, esta actitud se ve con mayor 
frecuencia en paciente que son portadores por primera vez de PPR los que han pasado de una etapa de inicialmente edéntulo a edéntulo quienes refieren menor aceptación al tratamiento ${ }^{15}$. El buen diseño y la implementación de controles posteriores permiten comprobar la eficacia de esta aparatología. Se debe proporcionar al paciente la mejor y mayor información sobre los beneficios e inconvenientes y promover las medidas de higiene $y$ de mantenimiento de las PPR 16 La utilización de una PPR conduce a cambios en la cantidad y calidad de la placa bacteriana, la ejecución de medidas adecuadas de higiene es fundamental para reducirlos ${ }^{17}$.

Existen cambios en la flora que se suceden en la cavidad bucal transcurrido uno a tres meses después de la instalación de la prótesis, debido a la mayor adhesión de los microorganismos en la superficie del acrílico. Esto se debe a que presenta cierto grado de porosidad, lo que proporciona sitios adecuados para la proliferación. En cambio, en la zona metálica, la proporción de microorganismos es menor, debido al pulido metalográfico que produce una superficie virtualmente carente de ranuras o rayas.

Existe una alta incidencia de caries dental y enfermedad periodontal en pacientes portadores de prótesis, donde la acumulación de placa dental sobre los dientes es evidente y está asociada con los ganchos de la prótesis o con la base de la misma $^{18}$.

La higiene del dispositivo protésico es de suma importancia, ya que el paciente debe comprender razonablemente las sugerencias y recomendaciones dada por el odontólogo. También hay que tener presente la acción de diversos desinfectantes sobre el armazón colado, los cuales pueden producir alteraciones en su superficie, se considera necesario el uso de estos agentes para la correcta eliminación de bacterias y otros microorganismos que se adhieren a las superficie de la prótesis ya que estudios sobre el mismo han demostrado la eficacia en la desinfección, donde los fenoles y los compuestos de amoniaco han demostrado ser los más efectivos, teniendo en cuenta que el cloro es el agente que mas alteraciones produce en la superficie metálica ${ }^{19}$.

Otro punto importante es explicar, instruir y verificar la maniobra de inserción y remoción del aparato protésico, debe hacerse sin forzar los retenedores para colocársela, para evitar desajustar o deformarlos. También explicar la manera de quitársela dejando claro las zonas donde debe hacer la tracción.

Todos los inconveniente devenidos por la utilización incorrecta del dispositivo protésico son nuevamente evaluados y corregido en los controles periódicos. En estos se evalúan los tejidos de soporte y la integridad de la estructura, el cumplimiento de las medidas de higiene y el mantenimiento de la PPR.

Una consecuencia poco frecuente pero evidenciada en varios trabajos de investigación realizados es la presencia de reacciones alérgicas de hipersensibilidad a los metales utilizados en Odontología, producto de la eliminación de pequeños iones metálicos. Un estudio In vitro sobre de aleaciones metálicas de Cromo cobalto molibdeno, observó la liberación de una mínima cantidad de iones cromo y una mayor liberación de cobalto produciendo un efecto contaminante que permite producir reacciones eczematosas sobre tés cutáneo ${ }^{20}$. Estas consecuencias alérgicas solo puede verificarse a través de los controles periódicos.

Si bien el lapso de 6 meses es adecuado para la mayoría de los pacientes, algunos pueden requerir evaluaciones más frecuentes. En casos de necesidad de incrementar la retención de los ganchos para asegurar una buena retención las visitas programadas dependerá del tipo de diseño o retenedor empleado. En clase I y II se tiene en cuenta que el soporte puede cambiar con el tiempo por lo cual la necesidad de reajuste de las bases es imperiosa. El balanceo y aflojamiento futuro de la prótesis se debe más al cambio de forma de la cresta que a la pérdida de retención, esto se debe solucionarse inmediatamente mediante un reajuste de la base. La pérdida de soporte tisular es gradual de manera que el paciente es incapaz de percibir los cambios. La necesidad de reajuste de la base es competencia del odontólogo debiendo ser detectada durante los durante el periodo de control. La pérdida de soporte en la base causa una pérdida de contacto oclusal y la separación del retenedor indirecto. El profesional lo debe descubrir durante los controles en la que se pone en evidencia por la rotación de la extensión distal alrededor de la línea de fulcrum. 


\section{Conclusiones}

El cumplimiento de las obligaciones y responsabilidades del paciente y del odontólogo deben quedar claramente definidas desde el inicio del tratamiento.

Es necesario implementar un sistema de controles inmediatos y mediatos de la PPR para poder evaluar la integridad y buen funcionamiento del dispositivo en el tiempo.

Los controles inmediatos se realizan a los 7 y 14 días de la fecha de instalación. En esta etapa se pueden presentar lesiones en la mucosa producto de la acción de los flancos sobre extendidos. Hay que localizar la zona extendida y reducir el grosor y/o longitud de los mismos.

La presencia de lesiones en los frenillos se debe a una invasión en la zona de movilidad del mismo, el que debe ser liberado inmediatamente.

Otro aspecto a tener en cuenta es la presencia de lesiones en la zona de asiento de la prótesis, esto puede deberse a un excesivo contacto de la misma.

Además es frecuente observar en paciente que reponen piezas dentarias posteriores, mordedura de lengua o carrillo, debido a que los mismos invaden la zona oclusal. Esta molestia desaparece con el tiempo debido a que se acomodan manteniéndose separadas de la zona oclusal.

Asimismo se indagará sobre la presencia de dolor en las piezas dentarias, que podría estar en relación con un contacto prematuro o por un excesivo ajuste del brazo retentivo del gancho, el cual será aliviado. El control con papel de articular y la eliminación a través de un desgaste selectivo reduce las molestias.

Otro punto es la presencia de nauseas y dificultad para hablar que con el tiempo irán disminuyendo. Para los controles mediatos el tiempo estipulado dependerá de cada paciente, se acepta en líneas generales un control a los tres meses de instalada la prótesis y después cada seis meses.

En los mismos se evaluará las estructuras de soporte y el funcionamiento de la prótesis. También se tendrá presente la inspección de todos los componentes de la PPR para evaluar la integridad de misma.

En este periodo se reforzara las medidas higiénicas y las instrucciones de mantenimiento y uso de las prótesis.

El paciente deberá ser informado que cualquier tratamiento adicional, reajuste, reparación o consulta que sea necesaria, el cual tendrá un costo adicional que deberá abonar y que formara parte de su tratamiento.

El cuidado y mantenimiento de la PPR exige controles regulares y continuos y no debe considerarse al tratamiento como permanente $y$ definitivo.

\section{Bibliografia}

I. ITOH H, BABA K, ARIDOME K, OKADA D, TOKUDA A, NISHIYAMA A, MIURA H. "Effect of direct retainer and major connector designs on RPD and abutment tooth movement dynamics". J Oral Rehabil. 2008 Nov; 35 (I I): 810 - 5. Epub 2008 May 9.

2. MC CORD JF, GREY NJ, WINSTANLEY RB, JOHNSON A. "A clinical overview of removable prostheses: 3. Principles of design for removable partial dentures." Dent Update. 2002 Dec; 29 (I0): 474 - $8 I$.

3. CARR A.B. y otros. "Mc Cracken Prótesis Parcial Removible" II ${ }^{a}$. Madrid. Elsevier. 2005.

4. DESPLATS EM. CALLIS EM. "Prótesis Parcial Removible y Sobredentaduras". Madrid. Elsevier. 2004.

5. LUK NK, WU VH, LIANG MB, CHEN YM, TIPKH, SMALES RJ. "Matematical analysis of occlusal rest design for cast removable partial denture". Eur J Prosthodont Restor Dent. 2007 Mar; 15 (I): 29 - 32.

6. PRESA N. "Mini incrustaciones adhesivas como apoyo en prótesis parcial removible". Revista de la Facultad de Odontologia de la UBA. 2000; 17 (44): $16-20$.

7. ALI M, WATERS NE, NAIRN RI, WEST $F$, SHERRIFF M. "A laboratory investigation of the role of guide planes in the retention of cast cobaltchromium alloy partial denture frameworks". J Dent. 200 I May; 29 (4): 291 - 9.

8. SALENBAUCH N. Prótesis parcial removible según concepto de base estable. Quintessenz. Febrero 2008; 21 (2): 85 - 95.

9. SALENBAUCH $N$.Prótesis parcial removible. Quintessenz. 2006; 57 (8): 839 - 49.

10. ANUSAVICE. "Philliss Ciencia de los Materiales Dentales" I I . Madrid. Elsevier. 2004.

II. COVA. N JL. "Biomateriales Dentales" España. Almorca. 2004.

12. RIVAS G MA. "Evaluación visual y radiográfica de discontinuidades en estructuras metálicas de pró- 
tesis parciales removibles". Acta Odontol. Venez v.39 n.2 Caracas. 2001

13. GREEN LK, HONDRUM SO. "The effect of design modifications on the torsional and compressive rigidity of U-shaped palatal major connectors." J Prosthet Dent. 2003 Apr; 89 (4): 400 $-7$.

14. PANNANNA AA, JOSHI SM, BHAT S, SHETTY P. "Evaluation of the polished surface characteristic of cobalt-chrome castings subsequent to various finishing and polishing techniques." Indian J Dent Res. 200 I Oct-Dec; 12 (4): 222 - 8.

15. BENEDICT R, KENNETH S. Nuevo grupo de pacientes para prótesis renovable: procedimiento para el tratamiento. J de Clínica en Odontología. 2000; 15 (3): I5 - 21

16. MCGIVNEY G, CARR A. "McCracken Prótesis Parcial Removible". I0a. Argentina. Editorial Medica Panamericana. 2004

17. VILA, V G. BARRIOS, C E. DHO, M S. PEREZ S. LANDAIDA M "Promoción y Educación para la Salud en adultos mayores". http://odn.unne.edu. ar/joex20065.pdf

18. ARDILA MEDINA, C.M... Efectos de la prótesis parcial removible sobre la salud periodontal. Avances en Periodoncia [online]. 2010, vol.22, n.2, pp. 77-83. ISSN 1699-6585. doi: 10.432I/ SI699-65852010000200003.

19. BAHANNAN SA, ABDEL-SALAM MM. "An invitro study of the effects of various disinfectants on prosthetic and surface materials." Saudi Med J. 2002 Apr; 23(4):396-9.

20. SUMMER B, FINK U, ZELLER R, RUEFF F, MAIER $S$, ROIDER G, THOMAS P. Patch test reactivity to cobalt-chromium-molybdenum alloy and stainless steel in metal-allergic patients in correlation to metal ion release. Contact Dermatitis. 2007 jul; 57 (I): 35 - 9. 